\title{
GLOBAL EXISTENCE OF WAVE MAPS IN $1+2$ DIMENSIONS WITH FINITE ENERGY DATA
}

\author{
Stefan Müller - Michael Struwe
}

Dedicated to Louis Nirenberg on the occasion of his 70th birthday

\section{Main result}

Let $N$ be a smooth, compact, $m$-dimensional Riemannian manifold, isometrically embedded in $\mathbb{R}^{d}$. A smooth map $u: \mathbb{R} \times \mathbb{R}^{2} \rightarrow N$ is called a wave map if

$$
u_{t t}-\Delta u \perp T_{u} N
$$

Let

$$
e(t, x):=\frac{1}{2}\left(\left|u_{t}\right|^{2}+|\nabla u|^{2}\right)
$$

denote the energy density. Smooth wave maps satisfy the energy identity

$$
E(t):=\int_{\{t\} \times \mathbb{R}^{2}} e(t, x) d x=\text { const. }
$$

In this note we show that the Cauchy problem for (1) admits a weak solution if the initial data have finite energy. For notational convenience we suppose $0 \in N$. We write $H^{1}$ for the Sobolev spaces $H^{1,2}\left(\mathbb{R}^{2}\right)$ or $H^{1,2}\left(\mathbb{R}^{2} ; \mathbb{R}^{d}\right)$.

1991 Mathematics Subject Classification. 35L70, 58E20, 58G16. 
Theorem 1.1. Suppose that $\left(u_{0}, u_{1}\right) \in H^{1} \times L^{2}$ and $u_{0}(x) \in N, u_{1}(x) \in$ $T_{u_{0}(x)} N$ a.e. Then there exists a global (forward) weak solution $u: \mathbb{R}_{0}^{+} \times \mathbb{R}^{2} \rightarrow N$ of the Cauchy problem

$$
\begin{gathered}
u_{t t}-\Delta u \perp T_{u} N \quad \text { in } \mathbb{R}^{+} \times \mathbb{R}^{2}, \\
u(0, \cdot)=u_{0}, \quad u_{t}(0, \cdot)=u_{1} .
\end{gathered}
$$

Moreover, u satisfies

$$
E(t) \leq E(0) \quad \forall t \geq 0
$$

and

$$
\left(\nabla u(t, \cdot), u_{t}(t, \cdot)\right) \rightarrow\left(\nabla u_{0}, u_{1}\right) \quad \text { in } L^{2} \times L^{2} \text {, as } t \rightarrow 0 .
$$

Here $u$ is called a weak solution of (1) if $u \in H_{\mathrm{loc}}^{1}\left(\mathbb{R}^{+} \times \mathbb{R}^{2}\right), u(t, x) \in N$ $\mathcal{L}^{3}$-a.e. in $\mathbb{R}^{+} \times \mathbb{R}^{2}$, if

$$
\int_{\mathbb{R}^{+} \times \mathbb{R}^{2}}\left\langle u_{t}, \varphi_{t}\right\rangle-\langle\nabla u, \nabla \varphi\rangle d x d t=0
$$

for all $\varphi \in H^{1}\left(\mathbb{R}^{+} \times \mathbb{R}^{2}\right)$ with $\varphi(z) \in T_{u(z)} N$ a.e. and compact support, and if

$$
u(t, \cdot) \rightarrow u_{0}, \quad u_{t}(t, \cdot) \rightarrow u_{1}
$$

in the sense of distributions (see Appendix A of [6] for the equivalence of various notions of a weak solution).

Existence of weak solutions was first established by Shatah [14] if $N=S^{k}$. His result was recently generalized by Freire [5] and Yi Zhou [15] to homogeneous spaces as targets. Short time existence and uniqueness for smooth data can be proved classically by energy methods. For a slightly modified problem that captures the essential difficulties of problem (1) Klainerman and Machedon [9], [10] established short time existence, uniqueness and continuous dependence for initial data in $H^{1+\delta} \times H^{\delta}, \delta>0$, through new Strichartz type estimates. This exploits the fact that (1) may be written explicitly as a system of hyperbolic differential equations with a particular null-form structure.

The key ingredient in our proof is a compactness result for wave maps under weak convergence ([7], [6]). Given this result a serious technical problem is to find suitable approximate problems for which existence is easy to prove. In this note we follow Yi Zhou [15] and use the viscous approximation

$$
u_{t t}-\Delta u-\varepsilon \Delta u_{t} \perp T_{u} N .
$$

Alternatively, one can use finite-difference approximations of (1) (see [12]).

To explain the compactness theorem it is useful to rewrite (1) as a first order system for $d u$ and the connection form of $T N$. We assume for the remainder of this section that $N$ is parallelizable. Let $\left(\bar{e}_{1}, \ldots, \bar{e}_{m}\right)$ be a smooth orthonormal frame of $T N$. Then, for a map $u: \mathbb{R}^{+} \times \mathbb{R}^{2} \rightarrow N$, the choice $e_{i}=\bar{e}_{i} \circ u$ yields an 
orthonormal frame $\left(e_{1}, \ldots, e_{m}\right)$ of the pullback bundle $u^{-1} T N$. Let $\theta_{i}:=\left\langle d u, e_{i}\right\rangle$ and let $\omega_{i j}$ denote the connection form given by $\omega_{i j}:=\left\langle d e_{i}, e_{j}\right\rangle$, where $\langle$,$\rangle is$ the scalar product in $\mathbb{R}^{d}$. The Lorentzian codifferential $\delta$ and the Lorentzian contraction act on 1-forms $\varphi=\varphi_{0} d t+\varphi_{1} d x^{1}+\varphi_{2} d x^{2}=\varphi_{\alpha} d x^{\alpha}, \psi=\psi_{\alpha} d x^{\alpha}$ by $\delta \varphi=\partial_{t} \varphi_{0}-\partial_{1} \varphi_{1}-\partial_{2} \varphi_{2}, \varphi \cdot \psi=-\varphi_{0} \psi_{0}+\varphi_{1} \psi_{1}+\varphi_{2} \psi_{2}$. With this notation equation (1) is equivalent to the system

$$
\delta \theta_{i}+\omega_{i j} \cdot \theta_{j}=0, \quad 1 \leq i \leq m .
$$

This is a straightforward calculation for smooth map; for the equivalence of weak solutions see Appendix A of [6].

Other frames $\tilde{e}_{i}$ of $u^{-1} T N$ can be obtained by the gauge transformation

$$
\widetilde{e}_{i}(x)=R_{i j}(x) e_{j}(x), \quad R(x)=\left(R_{i j}(x)\right) \in \mathrm{SO}(d) .
$$

This frame invariance can be exploited to (locally) choose a frame for which $\delta_{\text {eucl }} \omega_{i j}=\partial_{\alpha} \omega_{i j, \alpha}=0$. Using the identity $d \omega_{i j}=d e_{i} \wedge d e_{j}, \mathcal{H}^{1}$ estimates for Jacobians ([3]), $\mathcal{H}^{1}$-BMO duality ([4]), and concentration compactness ([11]) one then obtains the following compactness result ([6], Theorem 3.7).

For convenience we state this result in the periodic setting. The Campanato space $L^{2,1}\left(T^{3}\right)$ with norm

$$
\|f\|_{L^{2,1}}^{2}:=\sup _{z \in T^{3}} \sup _{0<R<1} \frac{1}{R} \int_{B(z, R)}|f|^{2} d \zeta
$$

consists of all $f \in L^{2}\left(T^{3}\right)$ that satisfy $\|f\|_{2,1}<\infty$. The semiarrow $\rightarrow$ denotes weak convergence.

TheOREM 1.2. Let $N$ be a parallelizable compact m-dimensional Riemannian manifold and suppose that the maps $v^{n}: T^{3} \rightarrow N$ satisfy

$$
v^{n} \rightarrow v \quad \text { in } H^{1}\left(T^{3} ; \mathbb{R}^{d}\right), \quad\left\|D v^{n}\right\|_{L^{2,1}} \leq C .
$$

Then there exist orthonormal frames $\left(\widetilde{e}_{1}^{n}, \ldots, \widetilde{e}_{m}^{n}\right)$ of $\left(v^{n}\right)^{-1} T N$ such that $\widetilde{e}_{i}^{n} \rightarrow$ $\widetilde{e}_{i}$ in $H^{1}\left(T^{3}, \mathbb{R}^{d}\right),\left(\widetilde{e}_{1}, \ldots, \widetilde{e}_{m}\right)$ is an orthonormal frame of $v^{-1} T N$ and

$$
\omega_{i j}^{n} \cdot \theta_{j}^{n} \rightarrow \omega_{i j} \cdot \theta_{j}+\nu_{i}
$$

in the sense of distributions, where $\nu_{i}$ is a Radon measure that satisfies

$$
\operatorname{supp} \nu_{i} \subset\left\{z \in T^{3}: \limsup _{R \rightarrow 0} \limsup _{n \rightarrow \infty}\left\|\chi_{B(z, R)} \theta^{n}\right\|_{L^{2,1}}>0\right\} .
$$

We will see that in this form, the convergence result can be directly applied to show weak convergence of solutions $\left(u_{\varepsilon}\right)$ of the Cauchy problem for (4) to (forward) weak solutions of (1), (2). 


\section{Regularized wave maps}

In this section we establish the global existence of solutions $u: \mathbb{R}_{0}^{+} \times \mathbb{R}^{2} \rightarrow N$ to the Cauchy problem for the regularized equation

$$
u_{t t}-\Delta u+\varepsilon \Delta u_{t} \perp T_{u} N .
$$

We do not assume that $N$ is parallelizable. Throughout this section we suppose

$$
0<\varepsilon \leq 1
$$

We first derive an expression for the normal component of $u_{t t}-\Delta u+\varepsilon \Delta u_{t}$ for an arbitrary (sufficiently smooth) map $u: \mathbb{R}^{+} \times \mathbb{R}^{2} \rightarrow N$. Let $\pi$ denote the nearest neighbour projection of a neighbourhood of $N$ to $N$ and let $P=\nabla \pi$. For $u \in N$, the linear map $P(u)$ is the orthogonal projection $\mathbb{R}^{d} \rightarrow T_{u} N$. Let $Q(u)=\operatorname{Id}-P(u)$ denote the projection on the normal space. We have (with summation over $i \in\{1,2\})$

$$
\begin{aligned}
P(u) u_{t t} & =\left(P(u) u_{t}\right)_{t}-P(u)_{t} u_{t} \\
& =u_{t t}-\nabla^{2} \pi(u)\left(u_{t}, u_{t}\right), \\
P(u) \Delta u & =\Delta u-\nabla^{2} \pi(u)\left(\partial_{i} u, \partial_{i} u\right), \\
P(u) \Delta u_{t} & =(P(u) \Delta u)_{t}-(P(u))_{t} \Delta u \\
& =\Delta u_{t}-\left(\nabla^{2} \pi(u)\left(\partial_{i} u, \partial_{i} u\right)\right)_{t}-\nabla^{2} \pi\left(u_{t}, \Delta u\right),
\end{aligned}
$$

and thus the normal component is given by

(8) $Q(u)\left(u_{t t}-\Delta u-\varepsilon \Delta u_{t}\right)=\nabla^{2} \pi(u)\left(\partial_{t} u, \partial_{t} u\right)-\nabla^{2} \pi(u)\left(\partial_{i} u, \partial_{i} u\right)$

$$
-\varepsilon\left(\nabla^{2} \pi(u)\left(\partial_{i} u, \partial_{i} u\right)\right)_{t}-\varepsilon \nabla^{2} \pi(u)\left(u_{t}, \Delta u\right)=: T(u) .
$$

In particular, for maps $u$ with values in $N$ equation (4) is equivalent to

$$
u_{t t}-\Delta u-\varepsilon \Delta u_{t}=T(u) .
$$

We consider Cauchy initial data

$$
u(0, \cdot)=u_{0}, \quad u_{t}(0, \cdot)=u_{1} .
$$

Lemma 2.1. Suppose that $\left(u_{0}, u_{1}\right) \in H^{2} \times H^{1}, u_{0}(x) \in N, u_{1}(x) \in T_{u_{0}(x)} N$ a.e. Then the initial value problem (9), (10) has a unique global solution $u$ : $\mathbb{R}_{0}^{+} \times \mathbb{R}^{2} \rightarrow N$ in the class

$$
X=H_{\mathrm{loc}}^{1}\left([0, \infty) ; H^{2}\right) \cap H_{\mathrm{loc}}^{1, \infty}\left([0, \infty) ; H^{1}\right) \cap H_{\mathrm{loc}}^{2}\left([0, \infty) ; L^{2}\right) .
$$

Moreover, the energy identity

$$
E(t)+\varepsilon \int_{0}^{t} \int_{\mathbb{R}^{2}}\left|\nabla u_{t}\right|^{2} d x d t=E(0)
$$

holds. 
Proof. This result appears already in Yi Zhou [15]. Since the proof of global existence may not be obvious to non-experts we sketch a proof of Lemma 2.1 .

Local existence is established by the usual fixed point argument. For convenience we scale

$$
v(t, x)=u(\varepsilon t, \varepsilon x)
$$

to achieve $\varepsilon=1$. To simplify the notation in the following we again write $u$ for $v$. Consider the spaces

$$
\begin{gathered}
X_{t, u_{0}, u_{1}}:=\left\{u \in H^{1}\left(0, t ; H^{2}\right) \cap H^{1, \infty}\left(0, t ; H^{1}\right) \cap H^{2}\left(0, t ; L^{2}\right):\right. \\
\left.u(0, \cdot)=u_{0}, u_{t}(0, \cdot)=u_{1}\right\}, \\
Y_{t}:=L^{2}\left(0, t ; L^{2}\right)
\end{gathered}
$$

with norms

$$
\begin{aligned}
\|u\|_{X_{t}}^{2}:= & \int_{0}^{t}\left[\|u\|_{H^{2}}^{2}(s)+\left\|u_{t}\right\|_{H^{2}}^{2}(s)+\left\|u_{t t}\right\|_{L^{2}}^{2}(s)\right] d s \\
& +\underset{s \in(0, t)}{\operatorname{ess} \sup }\left[\|u\|_{H^{2}}^{2}(s)+\left\|u_{t}\right\|_{H^{1}}^{2}(s)\right], \\
\|f\|_{Y_{t}}^{2}:= & \int_{0}^{t}\|f\|_{L^{2}}^{2}(s) d s .
\end{aligned}
$$

Here $\|u\|_{H^{2}}(s), \ldots$ denote the spatial norms at fixed times and we will abbreviate $\|u\|_{2}(s):=\|u\|_{L^{2}}(s)$, etc. and will suppress $s$ when no confusion can occur.

For $f \in Y_{t}$ the linear equation

$$
u_{t t}-\Delta u-\Delta u_{t}=f
$$

has a unique solution in $X_{t}$. Testing with $\Delta u_{t}$ we obtain the estimate

$$
\frac{d}{d t}\left(\left\|\nabla u_{t}\right\|_{2}^{2}+\|\Delta u\|_{2}^{2}\right)(s)+\left\|\Delta u_{t}\right\|_{2}^{2}(s) \leq\|f\|_{2}^{2}(s) \quad \text { for a.e. } s \in(0, t) .
$$

It follows that

$$
\begin{aligned}
& \underset{s \in(0, t)}{\operatorname{ess} \sup }\left(\left\|\nabla u_{t}\right\|_{2}^{2}+\|\Delta u\|_{2}^{2}\right)(s) \leq\|f\|_{Y_{t}}^{2}+\left\|u_{1}\right\|_{H^{1}}^{2}+\left\|u_{0}\right\|_{H^{2}}^{2}, \\
& \left\|\Delta u_{t}\right\|_{Y_{t}}^{2} \leq\|f\|_{Y_{t}}^{2}+\left\|u_{1}\right\|_{H^{1}}^{2}+\left\|u_{0}\right\|_{H^{2}}^{2} .
\end{aligned}
$$

In view of the identity $\left\|u_{t t}\right\|_{Y}=\left\|\Delta u+\varepsilon \Delta u_{t}+f\right\|_{Y}$, the Sobolev estimates (for $s \in(0, t))$,

$$
\begin{gathered}
\left\|u_{t}(s, \cdot)-u_{1}\right\|_{2} \leq C t^{1 / 2}\left\|u_{t t}\right\|_{Y_{t}}, \\
\left\|\nabla u(s, \cdot)-\nabla u_{0}\right\|_{2} \leq C t \underset{s \in(0, t)}{\operatorname{ess} \sup _{s}\left\|\nabla u_{t}\right\|_{2},} \\
\left\|u(s, \cdot)-u_{0}\right\|_{2} \leq C t \underset{s \in(0, t)}{\operatorname{ess} \sup _{s}}\left\|u_{t}\right\|_{2},
\end{gathered}
$$


and the identity $\left\|\nabla^{2} u\right\|_{2}=\|\Delta u\|_{2}$ we deduce that, for $t \in(0,1)$,

$$
\|u\|_{X_{t}} \leq C\left(1+t^{3 / 2}\right)\left(\|f\|_{Y_{t}}+\left\|u_{0}\right\|_{H^{2}}+\left\|u_{1}\right\|_{H^{1}}\right) .
$$

To proceed, we globally extend the map $\pi$-and hence the operator $T$ in (9) to an arbitrary, sufficiently smooth map $u: \mathbb{R}^{+} \times \mathbb{R}^{2} \rightarrow \mathbb{R}^{d}$ —as follows.

Let $2 \delta>0$ be the radius of a tubular neighbourhood $U_{2 \delta}(N)$ of $N$ such that the above projection $\pi$ is smooth and uniquely defined as a map $\pi: U_{2 \delta}(N) \rightarrow N$.

Let $\chi \in C^{\infty}(\mathbb{R})$ denote a function such that $\chi(s)=s$ for $s \leq \delta^{2}, \chi(s)=\frac{3}{2} \delta^{2}$ for $s \geq 2 \delta^{2}$, and $\chi^{\prime}(s) \geq 0$ for all $s$. The map $\varrho$ given by

$$
\varrho(u)=\chi\left(\frac{\operatorname{dist}^{2}(u, N)}{2}\right) \quad \text { for } u \in U_{2 \delta}(N)
$$

then extends to a smooth map on $\mathbb{R}^{d}$ with gradient

$$
\nabla \varrho(u)=u-\pi(u) \quad \text { for } u \in U_{\delta}(N) .
$$

Defining

$$
\bar{\pi}(u)=u-\nabla \varrho(u), \quad u \in \mathbb{R}^{d},
$$

we thus obtain the desired smooth extension of the nearest neighbour projection $\pi$ to a map $\bar{\pi}: \mathbb{R}^{d} \rightarrow \mathbb{R}^{d}$. In the following, we again write $\pi$ for $\bar{\pi}$. Observe that

$$
\pi(u)=u \quad \text { for } u \notin U_{2 \delta}(N) \text {; }
$$

hence

$$
\left|\nabla^{k} \pi(u)\right| \leq C_{k}=C_{k}(N) \text { for all } u \in \mathbb{R}^{d}, k \geq 1 .
$$

To establish short time existence of solutions of (9), (10) it suffices to show that the map $\mathcal{T}: u \rightarrow T(u)$ has the following properties:

$$
\mathcal{T} \text { maps bounded subsets of } X_{t, u_{0}, u_{1}} \text { to bounded subsets of } Y_{t} \text {; }
$$

$$
\|\mathcal{T}(u)-\mathcal{T}(v)\|_{Y_{t}} \leq C(R) t^{1 / 4}\|u-v\|_{X_{t}} \quad \text { for } t \leq 1,
$$

where $R=\max \left(\|u\|_{X_{t}},\|v\|_{X_{t}}\right)$.

To show boundedness of $\mathcal{T}$ note that

$$
|T(u)| \leq C\left(\left|u_{t}\right|^{2}+|\nabla u|^{2}+\left|u_{t}\right| \cdot|\nabla u|^{2}+|\nabla u| \cdot\left|\nabla u_{t}\right|+\left|u_{t}\right| \cdot|\Delta u|\right) .
$$

By Ladyzhenskaya's inequality and the identity $\left\|\nabla^{2} u\right\|_{2}=\|\Delta u\|_{2}$ we have, at fixed time,

$$
\begin{gathered}
(21) \quad\left\|u_{t}^{2}\right\|_{2}^{2} \leq\left\|u_{t}\right\|_{4}^{4} \leq C\left\|u_{t}\right\|_{2}^{2}\left\|\nabla u_{t}\right\|_{2}^{2}, \\
(22) \quad\left\||\nabla u|^{2}\right\|_{2}^{2} \leq\|\nabla u\|_{4}^{4} \leq C\|\nabla u\|_{2}^{2}\|\Delta u\|_{2}^{2}, \\
(23) \quad\left\|\left.u_{t}|\cdot| \nabla u\right|^{2}+\left|u_{t} \Delta u\right|\right\|_{2}^{2} \leq C\left\|u_{t}\right\|_{\infty}^{2}\left(1+\|\nabla u\|_{2}^{2}\right)\|\Delta u\|_{2}^{2}, \\
(24) \quad\left\||\nabla u| \cdot\left|\nabla u_{t}\right|\right\|_{2}^{2} \leq\|\nabla u\|_{4}^{2}\left\|\nabla u_{t}\right\|_{4}^{2} \leq C\|\nabla u\|_{2}\|\Delta u\|_{2}\left\|\nabla u_{t}\right\|_{2}\left\|\Delta u_{t}\right\|_{2},
\end{gathered}
$$


and hence

$$
\left\||\nabla u| \cdot\left|\nabla u_{t}\right|\right\|_{2}^{2} \leq \frac{C}{\delta}\|\nabla u\|_{2}^{2}\left\|\nabla u_{t}\right\|_{2}^{2}\|\Delta u\|_{2}^{2}+\delta\left\|\Delta u_{t}\right\|_{2}^{2} .
$$

In view of the estimate (see (31) below for a refinement)

$$
\left\|u_{t}\right\|_{\infty}^{2} \leq C\left\|u_{t}\right\|_{H^{1}}\left\|u_{t}\right\|_{H^{2}} \leq \frac{C}{\delta}\left\|u_{t}\right\|_{H^{1}}^{2}+\delta\left\|\Delta u_{t}\right\|^{2}
$$

it follows (with the choice $\delta=1$ above and in (25)) that

$$
\|\mathcal{T}(u)\|_{Y_{t}}^{2} \leq C\left(1+\|u\|_{X_{t}}^{6}\right) \quad \text { for } t \leq 1
$$

For future references we also note the finer estimate

$$
\begin{aligned}
\|\mathcal{T}(u)\|_{Y_{t}}^{2} \leq & C(\delta) t\left(1+\left[\sup _{s}\left(\|u\|_{H^{2}}+\left\|u_{t}\right\|_{H^{1}}\right)(s)\right]^{6}\right) \\
& +\delta\left\|\Delta u_{t}\right\|_{Y_{t}}^{2}\left(1+\sup _{s}\|u\|_{H^{2}}^{4}\right) \text { for all } t \in \mathbb{R}_{0}^{+} .
\end{aligned}
$$

The proof of the Lipschitz estimate (19) is similar with the following modifications. First, instead of the quadratic and cubic expressions in (21)-(24) one has to estimate similar expressions in $u$ and $w:=v-u$. Application of the Sobolev estimates in time (14)-(16) to $w$ yields the additional small factor of $t^{1 / 4}$ since $w_{0}=w_{1}=0$. Second, an additional term that can be estimated by

$$
C|w|\left(\left|u_{t}\right|^{2}+|\nabla u|^{2}+\left|u_{t}\right| \cdot|\nabla u|^{2}+|\nabla u| \cdot\left|\nabla u_{t}\right|+\left|u_{t}\right| \cdot|\Delta u|\right),
$$

plus a similar term with $u$ replaced by $v$, arises.

In view of (20)-(24) and the estimate

$$
\|w\|_{\infty}(s) \leq C\|\Delta w\|_{2}(s) \leq t^{1 / 2}\|w\|_{X_{t}}^{1 / 2}
$$

this term poses no additional difficulty.

Hence (18) and (19) hold, and (9), (10) has a solution $u:[0, \tilde{t}) \times \mathbb{R}^{2} \rightarrow \mathbb{R}^{d}$ up to a time $\widetilde{t}=\widetilde{t}\left(u_{0}, u_{1}\right)>0$. To see that $u$ takes values in $N$ observe that for short times $u$ is uniformly close to $N$ in view of the embedding $H^{1}\left(0, t ; H^{2}\right) \hookrightarrow$ $C^{0}\left(0, t ; C^{0}\right)$. Hence the projection $v:=\pi \circ u$ takes values in $N$. Moreover,

$$
\begin{aligned}
v_{t} & =\nabla \pi(u) u_{t} \\
v_{t t} & =\nabla \pi(u) u_{t t}+\nabla^{2} \pi(u)\left(u_{t}, u_{t}\right), \\
\Delta v & =\nabla \pi(u) \Delta u+\nabla^{2} \pi(u)\left(\partial_{i} u, \partial_{i} u\right), \\
\Delta v_{t} & =\nabla \pi(u) \Delta u_{t}+\nabla^{2} \pi(u)\left(u_{t}, \Delta u\right)+\left(\nabla^{2} \pi(u)\left(\partial_{i} u, \partial_{i} u\right)\right)_{t} .
\end{aligned}
$$

Using (9) we deduce that $w=v-u$ satisfies

$$
\begin{aligned}
w_{t t}-\Delta w-\varepsilon \Delta w_{t}= & (\nabla \pi \circ u) T(u) \\
= & {[(\nabla \pi \circ u)-(\nabla \pi \circ v)] T(u) } \\
& +(\nabla \pi \circ v)(T(u)-T(v))+(\nabla \pi \circ v) T(v) .
\end{aligned}
$$


Since $v$ takes values in $N$ it follows from $(8)$ that $(\nabla \pi \circ v) T(v)=0$. Now $w_{0}=w_{1}=0$ and thus by (17), (19) and (26),

$$
\begin{aligned}
\|w\|_{X_{t}} & \leq C\left[\left(\sup _{(0, t)}\|w\|_{\infty}\right)\|\mathcal{T}(u)\|_{Y_{t}}+\|\mathcal{T}(u)-\mathcal{T}(v)\|_{Y_{t}}\right] \\
& \leq C(R)\left(t^{1 / 2}+t^{1 / 4}\right)\|w\|_{X_{t}} \quad \text { for } t \leq 1
\end{aligned}
$$

where $R=\max \left(\|u\|_{X_{t}},\|v\|_{X_{t}}\right) \leq C\|u\|_{X_{t}}$. Hence $w \equiv 0$ on $(0, \widehat{t})$ for sufficiently small $\widehat{t}$. Thus for each $u_{0}, u_{1}$ as in Lemma 2.1 there exists a solution $u:(0, \widehat{t}) \times$ $\mathbb{R}^{2} \rightarrow N$ for some $\widehat{t}\left(u_{0}, u_{1}\right)>0$. By the usual continuation argument this solution can be extended to a maximal time interval $\left(0, t^{*}\right)$ and we will see that $t^{*}=\infty$ unless

$$
\limsup _{t \nearrow t^{*}}\|u\|_{H^{2}}(t)+\left\|u_{t}\right\|_{H^{1}}(t)=\infty .
$$

Indeed, if $\|u\|_{H^{2}}(t)+\left\|u_{t}\right\|_{H^{1}}(t)$ remains bounded by $C_{0}$ as $t \nearrow t^{*}$ then (13), (27) (with $\delta=1 /\left(2\left(1+C_{0}^{4}\right)\right)$ ) and (17) imply that $\left\|\Delta u_{t}\right\|_{Y_{t}}$ and $\|u\|_{X_{t}}$ also remain bounded as $t \nearrow t^{*}$. Hence $u(t, \cdot) \rightarrow \bar{u}_{0}$ in $H^{2}$ and $u_{t}(t, \cdot) \rightarrow \bar{u}_{1}$ in $L^{2}$ (and weakly in $H^{1}$ ) as $t \nearrow t^{*}$ and thus $\bar{u}_{0}$ takes values in $N$ and $\bar{u}_{1} \in T_{\bar{u}_{0}} N$. Therefore one can solve locally with initial data $\bar{u}_{0}, \bar{u}_{1}$ and thus extend the solution beyond $\left(0, t^{*}\right)$.

To establish global existence we use a Gronwall type estimate to show that (29) cannot hold for $t^{*}<\infty$.

Testing (9) with $u_{t} \in T_{u} N$ we obtain the energy identity

$$
\frac{1}{2}\left(\left\|u_{t}\right\|_{2}^{2}+\|\nabla u\|_{2}^{2}\right)(t)+\varepsilon \int_{0}^{t}\left\|\nabla u_{t}\right\|_{2}^{2}=E_{0} .
$$

We now return to the estimates (20)-(23) and use the Brezis-Waigner inequality for $u_{t}$ :

$$
\left\|u_{t}\right\|_{\infty} \leq C\left\|u_{t}\right\|_{H^{1}}\left[1+\ln ^{1 / 2}\left(1+\frac{\left\|u_{t}\right\|_{H^{2}}}{\left\|u_{t}\right\|_{H^{1}}}\right)\right] .
$$

In view of the estimate $a b \leq e^{a}+b \ln b$ (for $b>0$ ) we deduce that (for $0<\delta \leq 1$ )

$$
\begin{aligned}
\left\|u_{t}\right\|_{\infty}^{2}\|\Delta u\|_{2}^{2} \leq & C\left\|u_{t}\right\|_{H^{1}}^{2}\left[1+\ln \left(1+\frac{\left\|u_{t}\right\|_{H^{2}}}{\left\|u_{t}\right\|_{H^{1}}}\right)\right]\|\Delta u\|_{2}^{2} \\
\leq & C\left\|u_{t}\right\|_{H^{1}}^{2}\left[\left(1+\frac{\left\|u_{t}\right\|_{H^{2}}}{\left\|u_{t}\right\|_{H^{1}}}\right)+\|\Delta u\|_{2}^{2} \ln \|\Delta u\|_{2}^{2}\right] \\
\leq & C\left\|u_{t}\right\|_{H^{1}}^{2}+C\left\|u_{t}\right\|_{H^{1}}\left(\left\|u_{t}\right\|_{H^{1}}+\left\|\Delta u_{t}\right\|_{2}\right) \\
& +C\left\|u_{t}\right\|_{H^{1}}^{2}\|\Delta u\|_{2}^{2} \ln \|\Delta u\|_{2}^{2} . \\
\leq & \left(\delta\left\|\Delta u_{t}\right\|_{2}^{2}+\frac{C}{\delta}\left\|u_{t}\right\|_{H^{1}}^{2}\right)+C\left\|u_{t}\right\|_{H^{1}}^{2}\|\Delta u\|_{2}^{2} \ln \|\Delta u\|_{2}^{2} .
\end{aligned}
$$

If we let

$$
h(t):=\frac{1}{2}\left(\left\|\nabla u_{t}\right\|_{2}^{2}+\|\Delta u\|_{2}^{2}\right), \quad g(t):=\left\|\nabla u_{t}\right\|_{2}^{2},
$$


take $\delta=1 / 4$ in (32) as well as in (25) and denote by $C$ constants that only depend on $E_{0}$ we deduce from (20)-(25), (30), (32), (12) and (9) that

$$
h^{\prime} \leq C(g+C)\left(h\left(\ln ^{+} h+1\right)+C\right) .
$$

Since $\int_{2}^{\infty} \frac{d s}{1+s+s \ln s}=\infty$ and $\int_{0}^{t^{*}} g d t \leq E_{0}$ it follows that $h(t)$ remains bounded as $t \nearrow t^{*}$. This contradicts (29). Thus $t^{*}=\infty$, proving global existence.

To characterize the singular set $\operatorname{supp} \nu_{i}$ in Theorem 1.2 we will use the local energy inequality in Lemma 2.2 below. Due to the regularizing term we cannot expect finite speed of propagation but we show that the influence of points outside the backward light cone becomes exponentially small as $\varepsilon \rightarrow 0$.

Let

and for $t \leq t_{0}$ let

$$
\psi(s)= \begin{cases}\exp (s) & \text { if } s \leq 0 \\ 2-\exp (-s) & \text { if } s>0\end{cases}
$$

$$
\varphi_{\varepsilon}(t, x)=\psi\left(\left[(1+\sqrt{\varepsilon})\left(t_{0}-t\right)-\left|x-x_{0}\right|\right] / \sqrt{\varepsilon}\right) .
$$

LEMma 2.2. Let $u$ be the solution of (9), (10) in Lemma 2.1. Then, for $0<s<t<t_{0}$,

$$
\int_{\{t\} \times \mathbb{R}^{2}} \varphi_{\varepsilon} e d x \leq \int_{\{s\} \times \mathbb{R}^{2}} \varphi_{\varepsilon} e d x .
$$

Moreover, for all balls $B\left(z_{0}, r\right) \subset B\left(z_{0}, R\right) \subset \mathbb{R}^{+} \times \mathbb{R}^{2}$,

$$
\frac{1}{r} \int_{B\left(z_{0}, r\right)} e d z \leq \frac{C}{R} \int_{B\left(z_{0}, R\right)} e d z+C \exp \left(-\frac{R}{2 \sqrt{\varepsilon}}\right) E_{0} .
$$

Proof. We have, for a.e. $t$, in the sense of distributions in $\mathbb{R}^{2}$,

$$
\begin{aligned}
e_{t} & =\left\langle u_{t t}-\Delta u, u_{t}\right\rangle+\operatorname{div}\left\langle\nabla u, u_{t}\right\rangle \\
& =\left\langle\varepsilon \Delta u_{t}, u_{t}\right\rangle+\operatorname{div}\left\langle\nabla u, u_{t}\right\rangle \\
& =-\varepsilon\left|\nabla u_{t}\right|^{2}+\operatorname{div}\left(\varepsilon\left\langle\nabla u_{t}, u_{t}\right\rangle+\left\langle\nabla u, u_{t}\right\rangle\right)
\end{aligned}
$$

and thus, abbreviating $\varphi=\varphi_{\varepsilon}$,

$$
\begin{aligned}
\frac{d}{d t} \int_{\mathbb{R}^{2}} \varphi e & =\int_{\mathbb{R}^{2}}\left(\varphi e_{t}+e \varphi_{t}\right) d x \\
& \leq \int_{\mathbb{R}^{2}}\left(-\varepsilon\left|\nabla u_{t}\right|^{2} \varphi+\varepsilon\left|\nabla u_{t}\right| \cdot\left|u_{t}\right| \cdot|\nabla \varphi|+|\nabla u| \cdot\left|u_{t}\right| \cdot|\nabla \varphi|+e \varphi_{t}\right) d x \\
& \leq \int_{\mathbb{R}^{2}}\left(-\varepsilon\left|\nabla u_{t}\right|^{2} \varphi+\frac{\varepsilon^{3 / 2}}{2}\left|\nabla u_{t}\right|^{2}|\nabla \varphi|+\frac{\varepsilon^{1 / 2}}{2} u_{t}^{2}|\nabla \varphi|+e\left(|\nabla \varphi|+\varphi_{t}\right)\right) d x \\
& \leq \int_{\mathbb{R}^{2}}\left[\varepsilon\left|\nabla u_{t}\right|^{2}(\sqrt{\varepsilon}|\nabla \varphi|-\varphi)+e\left((1+\sqrt{\varepsilon})|\nabla \varphi|+\varphi_{t}\right)\right] d x \\
& \leq 0 \text { for a.e. } t .
\end{aligned}
$$


Since $t \rightarrow \int_{\mathbb{R}^{2}} \varphi e$ is absolutely continuous (in fact in $H_{\text {loc }}^{1}\left(t, t_{0}\right)$ ) this proves the first inequality. To establish the second estimate, note that we may assume $r \leq R / 16$ and that at the expense of increasing the constants we may replace balls by cylinders $C\left(z_{0}, r\right)=\left[t_{0}-r, t_{0}+r\right] \times B\left(x_{0}, r\right)$. It follows from (33) that, for $s \in[t+r-R / 4, t]$,

$$
\begin{aligned}
\int_{\{t\} \times B\left(x_{0}, r\right)} e d x & \leq \int_{\{s\} \times \mathbb{R}^{2}} e \psi\left(\left[(1+\sqrt{\varepsilon})(t-s)+r-\left|x-x_{0}\right|\right] / \sqrt{\varepsilon}\right) d x \\
& \leq \int_{\{s\} \times B\left(x_{0}, R\right)} 2 e d x+\int_{\{s\} \times\left(\mathbb{R}^{2} \backslash B\left(x_{0}, R\right)\right)} \exp \left(-\frac{R}{2 \sqrt{\varepsilon}}\right) e d x \\
& \leq \int_{\{s\} \times B\left(x_{0}, R\right)} 2 e d x+\exp \left(-\frac{R}{2 \sqrt{\varepsilon}}\right) E_{0} .
\end{aligned}
$$

Integration over $t \in\left[t_{0}-r, t_{0}+r\right]$ and over $s \in\left[t_{0}-\frac{1}{8} R, t_{0}-\frac{1}{16} R_{0}\right]$ yields the desired estimate for cylinders.

\section{Existence of wave maps}

Proof of Theorem 1.1. By a construction of Schoen and Uhlenbeck ([13], Section 4) there exist $u_{0 \varepsilon} \in C_{0}^{\infty}\left(\mathbb{R}^{2}, N\right)$ and $\widetilde{u}_{1 \varepsilon} \in C_{0}^{\infty}\left(\mathbb{R}^{2}, \mathbb{R}^{d}\right)$ such that

$$
u_{0 \varepsilon} \rightarrow u \quad \text { in } H^{1}, \quad \widetilde{u}_{1 \varepsilon} \rightarrow u_{1} \quad \text { in } L^{2} .
$$

Then $P\left(u_{0 \varepsilon}\right) \rightarrow P(u)$ boundedly a.e. and thus

$$
u_{1 \varepsilon}:=P\left(u_{0 \varepsilon}\right) \widetilde{u}_{1 \varepsilon} \rightarrow u_{1} \quad \text { in } L^{2} .
$$

By Lemma 2.1 there exists a global solution $u_{\varepsilon}: \mathbb{R}_{0}^{+} \times \mathbb{R}^{2} \rightarrow N$ of the Cauchy problem

$$
\begin{gathered}
u_{\varepsilon t t}-\Delta u_{\varepsilon}-\varepsilon \Delta u_{\varepsilon t} \perp T_{u_{\varepsilon}} N, \\
u_{\varepsilon}(0, \cdot)=u_{0 \varepsilon}, \quad u_{\varepsilon t}(0, \cdot)=u_{1 \varepsilon} .
\end{gathered}
$$

In view of the energy identity (30) there exists a sequence $\varepsilon_{n} \rightarrow 0$ such that $u^{n}:=u_{\varepsilon_{n}}$ satisfies

$$
\begin{array}{cl}
u^{n} \rightarrow u & \text { in } L_{\mathrm{loc}}^{2}\left(\mathbb{R}_{0}^{+} ; \mathbb{R}^{2}\right), \\
D u^{n} \stackrel{*}{\rightarrow} D u & \text { in } L^{\infty}\left(\mathbb{R}_{0}^{+} ; L^{2}\right), \\
\varepsilon \nabla u_{t}^{n} \rightarrow 0 & \text { in } L^{2}\left(\mathbb{R}_{0}^{+} \times \mathbb{R}\right) .
\end{array}
$$

We claim that $u$ is a weak wave map in $\mathbb{R}^{+} \times \mathbb{R}^{2}$. It suffices to check the assertion for every cube $Q^{\prime}=Q\left(z_{0}, r\right)=z_{0}+(-r, r)^{3}$ and we may assume $Q\left(z_{0}, 2 r\right) \subset \mathbb{R}^{+} \times \mathbb{R}^{2}$. Fix such a cube. We assume for convenience that $r=1 / 4$, the general case follows by scaling. By reflection across the planes $z^{\alpha}-z_{0}^{\alpha}= \pm 1 / 4$ and periodic extension we obtain maps $v^{n}: T^{3} \rightarrow N$ that satisfy 


$$
v_{\mid Q^{\prime}}^{n}=u^{n}, \quad\left\|D v^{n}\right\|_{L^{2}\left(\{t\} \times T^{2}\right)} \leq 2\left\|D u^{n}\right\|_{L^{2}\left(\{t\} \times Q\left(x_{0}, 1 / 4\right)\right)} \leq C .
$$

The last estimate implies that $D v^{n}$ is bounded in $L^{2,1}\left(T^{3}\right)$.

To proceed, we first make the additional assumption that $N$ is parallelizable. Let $\left(\widetilde{e}_{1}^{n}, \ldots, \widetilde{e}_{m}^{n}\right)$ be the frames of $\left(v^{n}\right)^{-1} T N$ whose existence is asserted in Theorem 1.2 and let $\theta_{i}^{n}=\left\langle d v^{n}, \widetilde{e}_{i}^{n}\right\rangle, \omega_{i j}^{n}=\left\langle d \widetilde{e}_{i}^{n}, \widetilde{e}_{j}^{n}\right\rangle$. Testing (22) with $\eta \widetilde{e}_{i}^{n}$ for $\eta \in C_{0}^{\infty}\left(Q^{\prime}\right)$ we obtain (cf. Section 1)

$$
\delta \theta_{i}^{n}+\omega_{i j}^{n} \cdot \theta_{j}^{n}=\operatorname{div}\left\langle\varepsilon \nabla u_{\varepsilon t}, \widetilde{e}_{i}^{n}\right\rangle-\left\langle\varepsilon \nabla u_{\varepsilon t}, \nabla \widetilde{e}_{i}^{n}\right\rangle
$$

in the sense of distributions in $Q^{\prime}$. It follows from (38)-(40) and Theorem 1.2 that

$$
\delta \theta_{i}+\omega_{i j} \cdot \theta_{j}=\nu_{i},
$$

in the sense of distributions in $Q^{\prime}$, where

$$
\operatorname{supp} \nu_{i} \subset S:=\left\{z \in Q^{\prime}: \limsup _{R \rightarrow 0} \limsup _{n \rightarrow \infty}\left\|\chi_{B(z, R)} D u^{n}\right\|_{L^{2,1}}>0\right\} .
$$

To finish the argument we show that $S$ has vanishing $H^{1,2}$ capacity and thus $\nu_{i}=0$ since the left hand side of (42) is in $H^{-1}+L^{1}$ (cf. [6] for further details). Indeed, passing to a further subsequence we may assume that

$$
\left|D u^{n}\right|^{2} \stackrel{*}{\rightarrow} \mu \quad \text { in } \mathcal{M}\left(Q^{\prime}\right) .
$$

Since $\varepsilon_{n} \rightarrow 0$ it then follows from the "monotonicity formula" (34) that

$$
\limsup _{n \rightarrow \infty}\left\|\chi_{B(z, R)} D u^{n}\right\|_{L^{2,1}} \leq C R^{-1} \mu(B(z, 2 R))
$$

and hence that

$$
S \subset\left\{z \in Q^{\prime}: \limsup _{R \rightarrow 0} \frac{1}{R} \mu(B(z, R))>0\right\} ;
$$

see [6] for the details.

Now the set on the right hand side is a countable union of sets of finite onedimensional Hausdorff measure and hence has vanishing $H^{1}$ capacity. Therefore

$$
\delta \theta_{i}+\omega_{i j} \cdot \theta_{j}=0
$$

as distributions in $Q^{\prime}$ and thus $u$ is a weak wave map in $\mathbb{R}^{+} \times \mathbb{R}^{2}$.

If $N$ is not parallelizable we use the fact that by [2] or [8], $N$ is a totally geodesic submanifold of a compact parallelizable Riemannian manifold $\widetilde{N}$, which in turn we may assume to be isometrically embedded in $\mathbb{R}^{d}$. Let $U \subset \mathbb{R}_{0}^{+} \times \mathbb{R}^{2}$ be open. Since the second fundamental forms of $N$ and $\widetilde{N}$ agree on $T N \times T N$, we have, for all $v \in H^{1}(U ; N)$ and all $\psi \in H^{1}\left(U ; v^{-1} T \widetilde{N}\right)$ with $\psi(v(x)) \perp T_{v(x)} N$,

$$
\left\langle\partial_{i} v, \partial_{i} \psi\right\rangle=0, \quad\left\langle\partial_{t} v, \partial_{t} \psi\right\rangle=0 .
$$


(It suffices to approximate $\partial_{i} v$ by $\left(P^{N} \circ v\right)\left(\varrho_{\varepsilon} * \partial_{i} v\right)$, the projection to $T_{v(x)} N$ of the standard mollification.) Let $\Pi(p)$ denote the orthogonal projection $T_{p} \widetilde{N} \rightarrow$ $T_{p} N$ and extend $\Pi(p)$ as the identity on $\left(T_{p} \widetilde{N}\right)^{\perp}$. Then

$$
\left\|\partial_{i}[(\Pi \circ v) \varphi]\right\|_{2} \leq C\left(\|\varphi\|_{\infty}+\|\nabla \varphi\|_{2}\right), \quad \forall \varphi \in\left(H^{1} \cap L^{\infty}\right)\left(U ; \mathbb{R}^{d}\right) .
$$

Now let $v^{n}: T^{3} \rightarrow N \hookrightarrow \widetilde{N}$ as above and apply Theorem 1.2 with $N$ replaced by $\widetilde{N}$. Let $\left(\widetilde{e}_{1}^{n}, \ldots, \widetilde{e}_{m^{\prime}}^{n}\right)$ be the corresponding frame of $\widetilde{N}$ and let $\theta_{i}^{n}=\left\langle d v^{n}, \widetilde{e}_{i}^{n}\right\rangle$, $\omega_{i j}^{n}=\left\langle d \widetilde{e}_{i}^{n}, \widetilde{e}_{j}^{n}\right\rangle$. As above we obtain, for $\eta \in C_{0}^{\infty}\left(Q^{\prime}\right)$,

$$
\left\langle\delta \theta_{i}^{n}+\omega_{i j}^{n} \cdot \theta_{j}^{n}, \eta\right\rangle=\int_{Q^{\prime}}\left\langle d v^{n}, d\left(\eta \widetilde{e}_{i}^{n}\right)\right\rangle,
$$

and application of (45) and (46) with $\varphi^{n}=\eta \widetilde{e}_{i}^{n}$ and $\Pi^{n}=\Pi \circ v^{n}$ yields

$$
\begin{aligned}
\int_{Q^{\prime}}\left\langle d v^{n}, d \varphi^{n}\right\rangle & =\int_{Q^{\prime}}\left\langle d v^{n}, d\left(\Pi^{n} \varphi^{n}\right)\right\rangle=\int_{Q^{\prime}}\left\langle v_{t t}^{n}-\Delta v^{n}, \Pi^{n} \varphi^{n}\right\rangle \\
& =\int_{Q^{\prime}}\left\langle\varepsilon_{n} \Delta v_{t}^{n}, \Pi^{n} \varphi^{n}\right\rangle \leq\left\|\varepsilon_{n} \nabla v_{t}^{n}\right\|_{2}\left\|\nabla\left(\Pi^{n} \varphi^{n}\right)\right\|_{2} \\
& \leq C \varepsilon_{n}\left\|\nabla v_{t}^{n}\right\|_{2} \rightarrow 0
\end{aligned}
$$

as $n \rightarrow \infty$. Hence

$$
\delta \theta_{i}^{n}+\omega_{i j}^{n} \cdot \theta_{j}^{n} \rightarrow 0
$$

in the sense of distributions, and as before we conclude that $v$ is a weak wave map (with values in $\widetilde{N}$ ). Since $v^{n} \rightarrow v$ in $L_{\text {loc }}^{2}$ the limit $v$ is a weak wave map with values in $N$.

While (44) was derived through the use of special frames the equation is frame-invariant (see [6], Appendix A for the weak setting) and hence holds in particular if $\theta$ and $\omega$ are defined with respect to the frame given by $e_{i}=\bar{e}_{i} \cdot u$, where $\left(\bar{e}_{i}\right)$ is a fixed frame of $N$. From now on we will work in this frame.

It remains to show that $u$ attains the correct initial values. We know that

$$
\begin{aligned}
& u^{n} \text { is bounded in } L_{\mathrm{loc}}^{\infty}\left(\mathbb{R}_{0}^{+} ; H^{1}\right), \\
& u_{t}^{n} \text { is bounded in } L^{\infty}\left(\mathbb{R}_{0}^{+} ; L^{2}\right) .
\end{aligned}
$$

Thus

$$
\left\|u^{n}(t, \cdot)-u_{0}^{n}\right\|_{2} \leq C t
$$

and, by (39) and the Aubin-Lions lemma,

$$
u^{n}(t, \cdot) \rightarrow u(t, \cdot) \quad \text { in } H^{1}, \text { for all } t \geq 0 .
$$

Letting $n \rightarrow \infty$ and $t \rightarrow 0$ in (47) and taking into account (38), (39) and (35) we deduce

$$
u(t, \cdot) \rightarrow u_{0} \quad \text { in } L^{2}, \quad u(t, \cdot) \rightarrow u_{0} \quad \text { in } H^{1} .
$$


To establish convergence of $u_{t}$ recall that the normal component of $u_{t t}^{n}$ is given by

$$
\left(\operatorname{Id}-P\left(u^{n}\right)\right) u_{t t}^{n}=\left(\nabla^{2} \pi\right)\left(u^{n}\right)\left(u_{t}^{n}, u_{t}^{n}\right) .
$$

To estimate the tangential components note that by (45),

$$
\begin{gathered}
\left\langle u_{t t}^{n}, e_{i}^{n}\right\rangle-\left\langle u_{t t}^{n}, \Pi^{n} e_{i}^{n}\right\rangle=\partial_{t}\left\langle u_{t}^{n}, e_{i}^{n}-\Pi e_{i}^{n}\right\rangle=0, \\
\left\langle\Delta u^{n}, e_{i}^{n}\right\rangle-\left\langle\Delta u^{n}, \Pi^{n} e_{i}^{n}\right\rangle=0
\end{gathered}
$$

and thus

$$
\left\langle u_{t t}^{n}, e_{i}^{n}\right\rangle=\operatorname{div}\left\langle\nabla u^{n}+\varepsilon \nabla u_{t}^{n}, \Pi^{n} e_{i}^{n}\right\rangle-\left\langle\nabla u^{n}+\varepsilon \nabla u_{t}^{n}, \nabla\left(\Pi^{n} e_{i}^{n}\right)\right\rangle .
$$

In combination with (39) and (40) it follows that

$$
\begin{aligned}
& u_{t}^{n} \text { is bounded in } L^{\infty}\left(\mathbb{R}_{0}^{+} ; L^{2}\right), \\
& u_{t t}^{n} \text { is bounded in }\left(L^{2}+L^{\infty}\right)\left(\mathbb{R}_{0}^{+} ;\left(L^{\infty} \cap H^{1}\right)^{*}\right) .
\end{aligned}
$$

Thus

$$
\left\|u_{t}^{n}(t, \cdot)-u_{t}^{n}(s, \cdot)\right\|_{2} \leq C\left(|s-t|^{1 / 2}+|s-t|\right)
$$

and by (38) and the Aubin-Lions lemma

$$
u_{t}^{n}(t, \cdot) \rightarrow u_{t}(t, \cdot) \quad \text { in } L^{2}, \text { for all } t \geq 0 .
$$

Letting $n \rightarrow \infty$ and $t \rightarrow 0$ in (48) and taking into account (36) we obtain $u_{t}(t, \cdot) \rightarrow u_{1}$ in $\left(L^{\infty} \cap H^{1}\right)_{\text {loc }}^{*}$ and therefore $u_{t}(t, \cdot) \rightarrow u_{1}$ in $L^{2}$, as $t \rightarrow 0$. Thus

$$
D u(t, \cdot) \rightarrow\left(\nabla u_{0}, u_{1}\right) \quad \text { in } L^{2}, \text { as } t \rightarrow 0,
$$

and, for every $t \geq 0$,

$$
D u^{n}(t, \cdot) \rightarrow D u(t, \cdot) \quad \text { in } L^{2} \text {, as } n \rightarrow \infty .
$$

The energy identity (30) yields

$$
\begin{aligned}
2 E(t) & =\|D u(t, \cdot)\|_{2}^{2} \leq \liminf _{n \rightarrow \infty}\left\|D u^{n}(t, \cdot)\right\|_{2}^{2} \\
& \leq \limsup _{n \rightarrow \infty}\left\|\left(\nabla u_{0}^{n}, u_{1}^{n}\right)\right\|_{2}^{2}=\left\|\left(\nabla u_{0}, u_{1}\right)\right\|_{2}^{2}=2 E(0) .
\end{aligned}
$$

Thus

$$
\limsup _{t \rightarrow 0}\|D u(t, \cdot)\|_{2}^{2} \leq\left\|\left(\nabla u_{0}, u_{1}\right)\right\|_{2}^{2}
$$

and hence strong convergence holds in (49). The proof of Theorem 1.1 is finished. 


\section{Concluding remarks}

A major open problem is the uniqueness of wave maps with finite energy in $1+2$ dimensions. Uniqueness in the class of all weak solutions would imply the energy identity $E(t)=E(0)$ for all $t \geq 0$, since otherwise time reversal would yield a weak solution for which the energy increases and which would thus be different from the solution constructed above. From the energy identity and (50), (51) one easily deduces that $D u^{n} \rightarrow D u$ in $L^{2}$ for the above approximations (this implies the local energy inequality for $u$ ) as well as continuity of the map $\mathbb{R}_{0}^{+} \rightarrow L^{2}$ given by $t \mapsto D u(t, \cdot)$. In particular, concentration of energy would be impossible. It is, however, widely believed that such a phenomenon may occur, as it does, for instance, for the harmonic heat flow $u_{t}-\Delta u \perp T_{u} N$ in $1+2$ dimensions (see [1]). Hence, uniqueness is only expected to hold in a more restricted class, defined by conditions such as monotonicity of the energy or local energy inequalitites.

Existence of solutions that enjoy such additional properties remains an open problem. On the other hand, the solutions constructed here still enjoy the compactness property originally established only for smooth solutions ([7], [6]). The main point is that in view of (34) the solutions constructed above satisfy, for $B\left(z_{0}, r\right) \subset B\left(z_{0}, R\right) \subset \mathbb{R}^{+} \times \mathbb{R}^{2}$,

$$
\frac{1}{r} \int_{B\left(z_{0}, r\right)}|D u|^{2} \leq \frac{C}{R} \mu\left(\overline{B\left(z_{0}, R\right)}\right),
$$

where $\mu$ is the Radon measure in (43). If $\left\{u^{l}\right\}$ is a sequence of such solutions with uniformly bounded energy and $u^{l} \rightarrow u$ in $L_{\text {loc }}^{2}\left(\mathbb{R}^{+} \times \mathbb{R}^{2}\right)$ then, after passage to a subsequence, we may assume $\mu^{l} \stackrel{*}{\rightarrow} \widetilde{\mu}$ and

$$
\limsup _{l \rightarrow \infty}\left\|\chi_{B\left(z_{0}, r\right)} D u^{l}\right\|_{L^{2,1}} \leq \frac{C}{R} \widetilde{\mu}\left(\overline{B\left(z_{0}, 3 R\right)}\right) .
$$

It now follows from Theorem 1.2 as above that $u$ is a weak wave map.

\section{REFERENCES}

[1] K. C. Chang, W. Y. Ding and R. G. Ye, Finite-time blow-up of the heat flow of harmonic maps from surfaces, J. Differential Geom. 36 (1992), 507-515.

[2] D. Christodoulou and A. S. Tahvildar-Zadeh, On the regularity of spherically symmetric wave maps, Comm. Pure Appl. Math. 46 (1993), 1041-1091.

[3] R. Coifman, P.-L. Lions, Y. Meyer and S. Semmes, Compensated compactness and Hardy spaces, J. Math. Pures Appl. 72 (1993), 247-286.

[4] C. Fefferman and E. M. Stein, $H^{p}$ spaces of several variables, Acta Math. 129 (1972), 137-193.

[5] A. FreIRE, Global weak solutions to the wave map system to compact homogeneous spaces, preprint. 
[6] A. Freire, S. Müller And M. Struwe, Weak compactness of wave maps and harmonic maps, Ann. Inst. H. Poincaré Anal. Non Linéaire (to appear).

[7] Weak convergence of wave maps from $(1+2)$-dimensional Minkowski space to Riemannian manifolds, Invent. Math. (to appear).

[8] F. HÉLEIN, Régularité des applications faiblement harmoniques entre une surface et une variété riemannienne, C. R. Acad. Sci. Paris Sér. I Math. 312 (1991), 591-596.

[9] S. Klainerman and M. Machedon, Space-time estimates for null forms and the local existence theorem, Comm. Pure Appl. Math. 46 (1993), 1221-1268.

[10] Smoothing estimates for null forms and applications, Internat. Math. Res. Notes 4 (1994), 383-389.

[11] P.-L. Lions, The concentration compactness principle in the calculus of variations, the limit case, part II, Rev. Mat. Iberoamericana 12 (1985), 45-121.

[12] S. Müller, M. Struwe And V. Šverák, in preparation.

[13] R. Schoen and K. Uhlenbeck, Boundary regularity and the Dirichlet problem for harmonic maps, J. Differential Geom. 18 (1983), 253-268.

[14] J. SнAтAн, Weak solutions and development of singularities in the SU(2) $\sigma$-model, Comm. Pure Appl. Math. 41 (1988), 459-469.

[15] Y. ZHOU, Global weak solutions for $1+2$ dimensional wave maps into homogeneous spaces, preprint, 1995.

Manuscript received June 5, 1996

S. MÜller and M. Struwe

Department of Mathematics

ETH Zürich

Rämistr. 101

CH-8092 Zürich, SWITZERLAND

E-mail address: sm@mis.mpg.de, struwe@math.ethz.ch 